\title{
Spider web-inspired acoustic metamaterials
}

\author{
Marco Miniaci, ${ }^{1}$ Anastasiia Krushynska, ${ }^{2}$ Alexander B. Movchan, ${ }^{3}$ Federico Bosia, ${ }^{2, a)}$ \\ and Nicola M. Pugno $4,5,6, a)$ \\ ${ }^{1}$ Laboratoire Ondes et Milieux Complexes, University of Le Havre, UMR CNRS 6294, 76600 Le Havre, France \\ ${ }^{2}$ Department of Physics, University of Torino, 10125 Torino, Italy \\ ${ }^{3}$ Department of Mathematical Sciences, University of Liverpool, L69 3BX Liverpool, United Kingdom \\ ${ }^{4}$ Laboratory of Bio-Inspired and Graphene Nanomechanics, Department of Civil, Environmental and \\ Mechanical Engineering, University of Trento, 38123 Trento, Italy \\ ${ }^{5}$ Center for Materials and Microsystems, Fondazione Bruno Kessler, 38123 Povo, Italy \\ ${ }^{6}$ School of Engineering and Materials Science, Queen Mary University of London, London E1 4NS, \\ United Kingdom
}

(Received 17 June 2016; accepted 5 August 2016; published online 19 August 2016)

Spider silk is a remarkable example of bio-material with superior mechanical characteristics. Its multilevel structural organization of dragline and viscid silk leads to unusual and tunable properties, extensively studied from a quasi-static point of view. In this study, inspired by the Nephila spider orb web architecture, we propose a design for mechanical metamaterials based on its periodic repetition. We demonstrate that spider-web metamaterial structure plays an important role in the dynamic response and wave attenuation mechanisms. The capability of the resulting structure to inhibit elastic wave propagation in sub-wavelength frequency ranges is assessed, and parametric studies are performed to derive optimal configurations and constituent mechanical properties. The results show promise for the design of innovative lightweight structures for tunable vibration damping and impact protection, or the protection of large scale infrastructure such as suspended bridges. Published by AIP Publishing. [http://dx.doi.org/10.1063/1.4961307]

Many natural materials display outstanding properties that can be attributed to their complex structural design, developed in the course of millions of years of evolution. ${ }^{1-3}$ Particularly fascinating are spider silks, which exhibit unrivalled strength and toughness when compared to most materials. $^{4-7}$ Previous studies have revealed that mechanical performance of spider webs is not only due to the remarkable properties of the silk material, but also to an optimized architecture that is adapted to different functions. ${ }^{8,9}$

Structural behaviour of orb spider webs has been extensively analyzed under quasi-static ${ }^{6,8,10}$ and dynamic ${ }^{11,12}$ loading conditions. However, the spider-web structure has yet to be exploited for the design of phononic structures. These are usually periodic composites capable of inhibiting the propagation of elastic waves in specific frequency ranges called band gaps. This unique ability opens a wide range of application opportunities, such as seismic wave insulation, ${ }^{13}$ noise reduction, ${ }^{14}$ sub-wavelength imaging and focusing, ${ }^{15}$ phonon transport, ${ }^{16}$ strain-dependent thermal conductivity, ${ }^{17}$ acoustic cloaking, ${ }^{18}$ and thermal control. ${ }^{19}$ In phononic structures, band gaps are induced by either Bragg scattering from periodic inhomogeneities ${ }^{20}$ or by local resonances. ${ }^{21}$ The latter are commonly achieved by employing heavy constituents. ${ }^{21-24}$ Recently, it has been found that hierarchically organized continuous ${ }^{25}$ or lattice-type ${ }^{26,27}$ structures exhibit band gaps due to the two mentioned mechanisms. From this perspective, a spider web-inspired, lattice-based elastic metamaterial seems to be another promising alternative to simultaneously control wave propagation at multi-scale frequencies. In this letter, we design a metamaterial inspired by

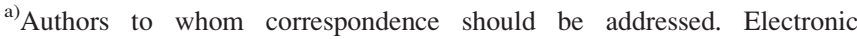
addresses: federico.bosia@unito.it and nicola.pugno@unitn.it
}

the Nephila orb web architecture and analyze the dynamics of elastic waves propagating therein, with the aim of obtaining improved structures compared to simple lattices. ${ }^{28}$

We consider a spider web-inspired metamaterial in the form of an infinite in-plane lattice modeled by periodically repeating representative unit cells in a square array. The primary structure of the unit cell is a square frame with supporting radial ligaments (Fig. 1(a)). The ligaments intersect the frame at right-angle junctions acting as "hinge" joints (square junctions in Fig. 1(a)). The secondary frame is defined by a set of equidistant circular ligaments (or ring resonators) attached to the radial ligaments by hinge joints, in the following called "connectors" to distinguish them from the joints in the first frame (Fig. 1(b)). The geometry of the metamaterial is completely defined by 5 parameters: unit cell pitch $a$, size of square joints $b$, thickness of radial and circular ligaments $c$, number of ring resonators $N$, and radius of a ring resonator $R_{N}$. We initially consider $a=1 \mathrm{~m}, b=0.04 \cdot a, c=0.01 \cdot a$, $N=7$, and $R_{N}=0.1 \cdot a \cdot(N+1) / 2$. The material properties
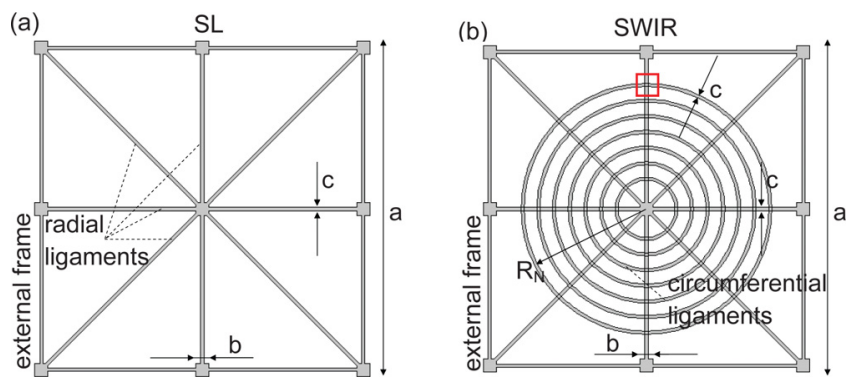

FIG. 1. (a) Bearing frame and (b) spider web-inspired unit cells for latticetype metamaterials. 
of the primary and secondary frames correspond to the parameters of dragline $\left(E_{d}=12 \mathrm{GPa}, \nu_{d}=0.4, \rho_{d}=1200 \mathrm{~kg} / \mathrm{m}^{3}\right)$ and viscid $\left(E_{v}=1.2 \mathrm{GPa}, \nu_{v}=0.4, \rho_{v}=1200 \mathrm{~kg} / \mathrm{m}^{3}\right)$ silks of the Nephila orb spider web, ${ }^{9}$ respectively. Material properties of the connectors can assume dragline or viscid silk values. The propagation of elastic waves is investigated numerically by using the Finite Element commercial package COMSOL Multiphysics. Wave dispersion in infinite lattices is studied by applying the Bloch conditions ${ }^{23}$ at the unit cell boundaries and performing the frequency modal analysis for wavenumbers along the borders $Г X M$ of the first irreducible Brillouin zone. $^{29}$

First, we study small-amplitude elastic waves propagating in an infinite structure formed by the primary frame unit cell (Fig. 1(a)), called "regular lattice" metamaterial. Fig. 2(a) shows band diagrams for the regular lattice as a function of reduced wave vector $k^{*}=\left[k_{x} a / \pi ; k_{y} a / \pi\right]$. The color scale, here and in other diagrams, shows polarization of waves propagating along the $x$ direction that varies from pure shear (blue) to pure longitudinal (red). Up to $400 \mathrm{~Hz}$, there is one negligible band gap around $80 \mathrm{~Hz}$. The band structure exhibits localized modes at various frequencies represented by (almost) flat bands. Analysis of the vibration forms reveals that the motion is localized within the radial ligaments, which are mainly subjected to flexural deformation (Fig. 2(e)).

Next, the circular elements are introduced to analyze the wave dispersion in a spider web-inspired metamaterial (Fig. 1(b)). Here, we explore three possibilities: (1) the circular ligaments have the same material properties as the radial ligaments (dragline silk); (2) the circular ligaments are made of viscid silk, while connectors of radial and circular ligaments have the properties of dragline silk, and (3) both the circular ligaments and the connectors are made of viscid silk. This allows evaluating the influence of material parameters on the performance in the spider-web structures. Fig. 2(b) shows the band diagram for the metamaterial made of the dragline silk with a complete band gap at frequencies from 346.5 to $367.4 \mathrm{~Hz}$, which is shaded in light gray. As the band gap bounds are formed by non-flat curves and the whole unit cell is involved in the motion at the band gap bound (Fig. 2(f)), this band gap is not due to local resonances. Also, the band gap cannot be induced by Bragg scattering, as it is located at least twice below the frequencies at which a half-wavelength of either longitudinal $(2314 \mathrm{~Hz})$ or shear $(945 \mathrm{~Hz})$ waves in the silk is equal to the unit cell size. Further analysis of the band gap origin is beyond the scope of this letter, since we are focusing on a spider web-inspired structure with different mechanical properties for radial and circular ligaments. ${ }^{8,9}$ Another remarkable feature of the band structure in Fig. 2(b) is the smaller number of localized modes compared to Fig. 2(a), which may be explained with the elimination of local resonances due to the coupling between motions in radial and circular ligaments.

By assigning viscid silk material properties to ring resonators, two band gaps appear in Figs. 2(c) and 2(d), regardless of the material properties of the connectors joining radial and circular ligaments. Due to the compliant behaviour of the resonators, the band gaps are located at lower frequencies compared to those in Fig. 2(b). These are so-called hybridization band gaps induced by local resonances, since (a)
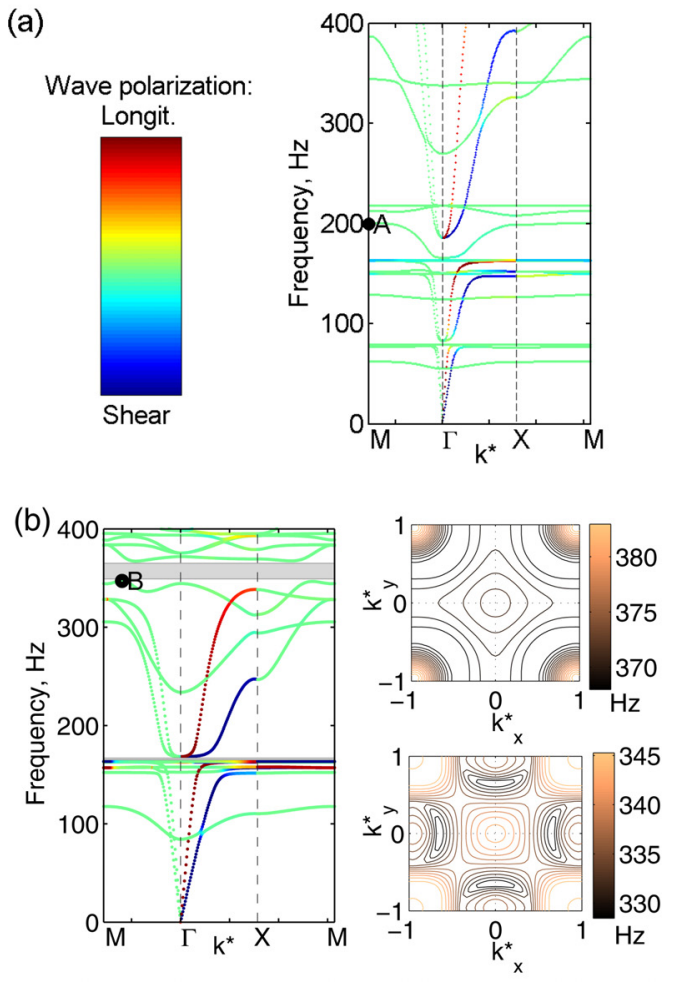
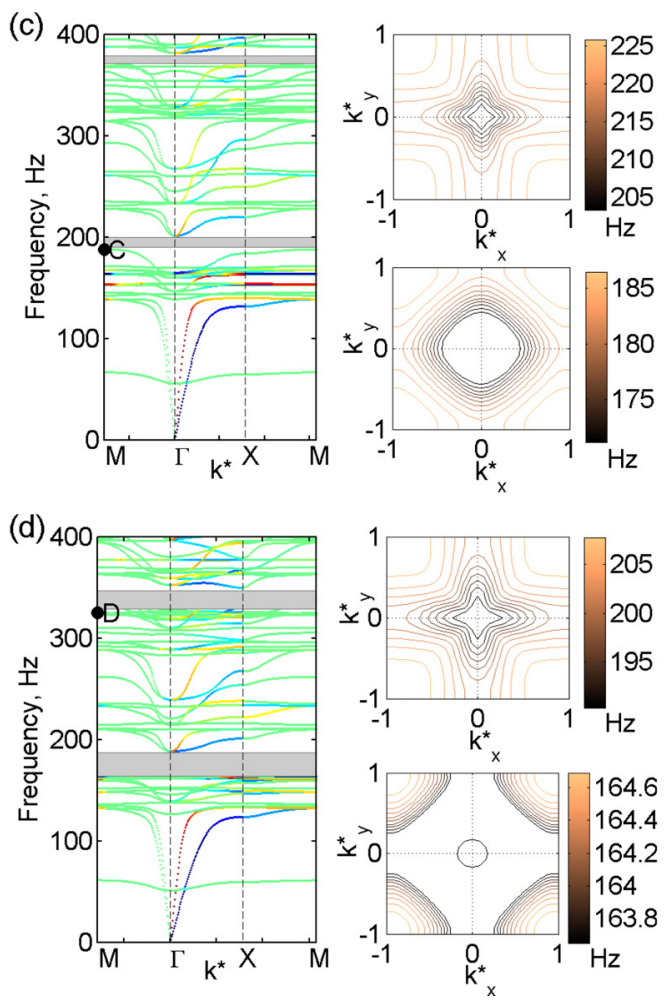

(g)
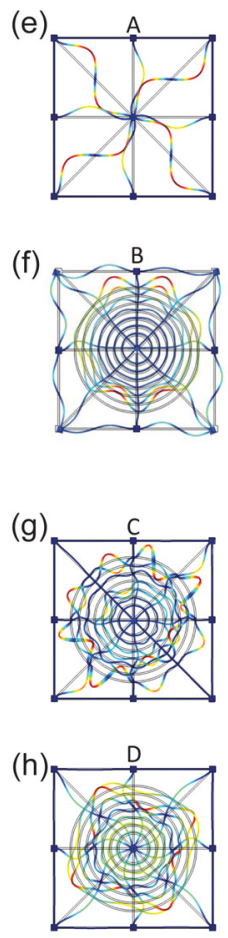

FIG. 2. (a) Band structure for the regular lattice. (b)-(d) Band structures and contour plots of dispersion surfaces at the upper and lower BG boundaries for spider web-inspired lattices: (b) stiff ring resonators (dragline silk), (c) compliant ring resonators (viscid silk) and rigid connectors (dragline silk), and (d) compliant ring resonators and connectors. Band gaps are shaded in gray and the color of pass bands represents the mode polarization ranging from pure shear (blue) to pure longitudinal (red). (e)-(h) Mode shapes referring to points A, B, C, and D of the dispersion diagrams. 
the lower bounds are formed by flat curves representing localized motions (Figs. 2(g) and 2(h)), and the Bloch wave vector $k^{*}$ changes by $\pi$ inside each band gap. ${ }^{31}$ When the ring resonators and the connectors have the same mechanical properties (viscid silk), the band gaps are shifted to lower frequencies due to a more compliant behaviour of the connectors (compare Figs. 2(c) and 2(d)).

To investigate the wave directionality, we evaluate dispersion surfaces for all directions within the first Brillouin zone. The results are shown as contour plots for pass bands at the band gap bounds (bottom and top figures on the right of each band diagram in Fig. 2). The color scales represent the values at which frequency cuts are performed. The contour plots reveal preferred directions of propagation at $\theta=0^{\circ}$ $\left(\theta=90^{\circ}\right)$ that indicates strong anisotropy in the wave dispersion near the band gaps, as in other phononic structures. ${ }^{30}$

Another peculiarity of the band diagrams in Figs. 2(c) and 2(d) in comparison to Figs. 2(a) and 2(b) is a larger number of localized modes. In the former case, these modes are associated with standing waves mostly dominated by high inertia of the resonators (Figs. 2(g) and 2(h)). If the connectors between radial and circular ligaments have the same material properties as the ring resonators (the closest configuration to a real spider web), it appears that the standing waves may be associated with the resonators only.

The natural frequencies $\omega_{n}$ for non-axisymmetric inplane flexural vibrations of these resonators can be expressed $^{32}$ in closed form as: $\omega_{n}=k \frac{n\left(n^{2}-1\right)}{R^{2} \sqrt{n^{2}+1}}$, with $n>1$. Here, $R$ stands for the radius of a ring resonator, and $k$ is a dimensional constant that depends on the elastic modulus of the ring resonator, the mass density, and its cross-section. Vibrational modes for several values of $n$ are shown in the supplementary material. However, this analytical solution does not describe the dynamics of spider-web lattice systems satisfactorily, since their response is governed by the entire structure and not the individual decoupled resonators (see the supplementary material for details). More insight into the wave dynamics in the proposed metamaterials is achieved by analyzing the mode transformations for varying geometrical and mechanical parameters (see the supplementary material). Next, we vary the stiffness of ring resonators by choosing

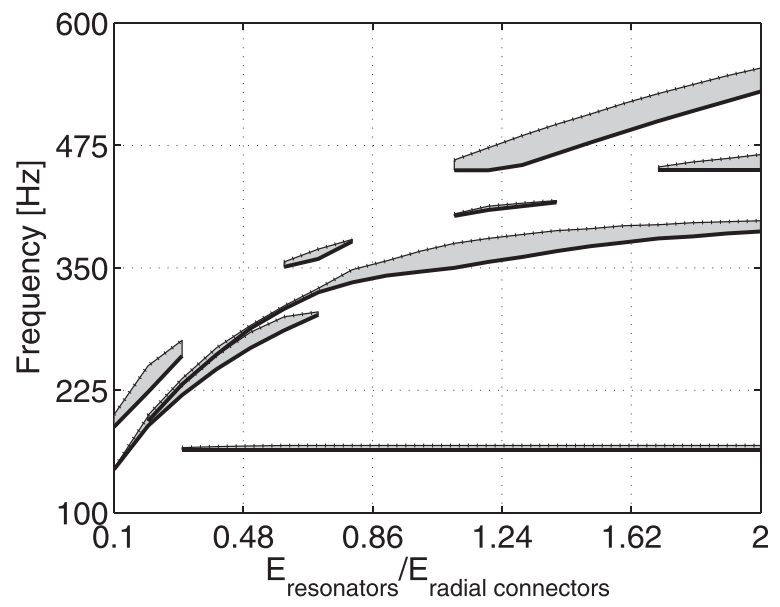

FIG. 3. Band gap frequencies for the lowest band gaps as functions of ratio $E_{r r} / E_{r l}\left(E_{r r}=12 \mathrm{GPa}\right)$.
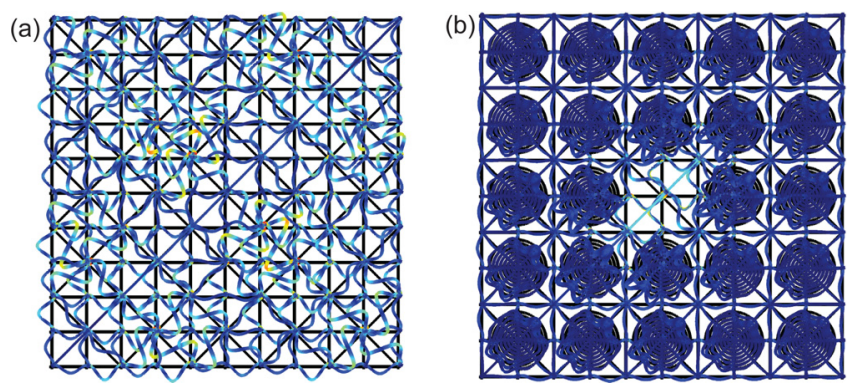

FIG. 4. Frequency domain in-plane displacements (scaled by 45000 ) for a point excitation of frequency $186 \mathrm{~Hz}$ applied at an angle $\pi / 4$ with respect to the horizontal axis in (a) a regular-lattice structure and (b) spider webinspired system with viscid-silk ring resonators. Red and blue colors indicate maximum and minimum displacements, respectively.

intermediate values between those of dragline and viscid silks. The overall band diagrams resemble those shown in Figs. 2(c) and 2(d). Thus, we focus our attention only on the band gaps. Fig. 3 shows band gap frequencies versus ratios $E_{r r} / E_{r l}$, where $E_{r r}=12 \mathrm{GPa}$ and $E_{r l}$ are the stiffnesses of the ring resonators and radial ligaments, respectively. In general, as the stiffness of the ring resonators increases, inhibited frequency ranges are translated towards higher frequencies, except the lowest band gap around $150 \mathrm{~Hz}$ with frequencies independent of the mechanical parameters of the resonators.

Now we investigate the transmission in finite-size spider-web inspired structures. The analysed model comprises 25 unit cells placed in a square array with traction-free boundary conditions. The structure is excited at the central point by applying harmonic in-plane displacement at a frequency of $186 \mathrm{~Hz}$ (within a band gap) at an angle of $\pi / 4$ with respect to the horizontal axis. Fig. 4 presents frequencydomain responses (scaled by a factor of 45000 ) in terms of in-plane displacements for two structures formed by the regular and spider-web lattice unit cells with viscid silk ring resonators. Maximum and minimum values of displacements are shown in red and dark blue, respectively. Notice that all of the regular-lattice structure vibrates (Fig. 4(a)), while the spider web-inspired system is capable of strongly attenuating vibrations after a few unit cells (Fig. 4(b)). A similar behaviour is observed for other excitation frequencies within the band gaps. These results confirm the predictions derived from the wave dispersion analysis. Fig. 4(b) suggests as an application the generation of a defect mode in a cluster with localized vibrations around its center for efficient wave attenuation at desired frequencies.

In summary, we have numerically studied the propagation characteristics of elastic waves in regular and spider web-inspired beam lattices, based on the Nephila orb web architecture. Our results indicate that these lattices possess locally resonant band gaps induced by either ring-shaped resonators or parts of the bearing frame. Dispersion analysis reveals strong anisotropic dynamics of spider-web lattices and the mixed character of localized modes. The band gaps can be easily tuned in a wide range of frequencies by varying the mechanical properties or the number of the resonators, or even the properties of the connectors between resonators and the frame. Despite the fact that the ring resonators are responsible for the generation of band gaps, their eigenfrequencies 
cannot be directly used to predict the band gap bounds, since the overall structure plays an important role in their formation. Though lattice systems with locally resonant band gaps have already been reported, ${ }^{26,27}$ this study shows that spider web-inspired lattice metamaterials are particularly efficient in inducing low-frequency band gaps despite being light-weight. Also, they possess more parameters to tune the band gaps to desired frequencies and are easier to manipulate/manufacture compared to hierarchically organized lattice structures. Thus, results from this study can inspire further designs of lightweight and robust metamaterial structures with tunable properties. This work also suggests an advanced functionality for spider webs and future applications for the corresponding metamaterials and metastructures, e.g., for earthquake protection of suspended bridges.

See supplementary material for more details.

M.M. is funded by the EU H2020 Grant No. 658483. A.K. is funded by FP7 Grant No. 609402-2020. N.M.P. is funded by ERC Grants 279985 and 693670, and the EU Graphene Flagship (WP 14 Polymer Nanocomposites, No. 696656). F.B. is funded by ERC No. 279985.

${ }^{1}$ H. Gao, B. Ji, I. L. Jaeger, E. Arzt, and P. Fratzl, "Materials become insensitive to flaws at nanoscales: Lessons from nature," Proc. Natl. Acad. Sci. U.S.A. 100, 5597-5600 (2003).

${ }^{2}$ J. Aizenberg, J. C. Weaver, M. S. Thanawala, V. C. Sundar, D. E. Morse, and P. Fratzl, "Skeleton of Euplectella sp.: Structural hierarchy from the nanoscale to the macroscale," Science 309, 275-278 (2005).

${ }^{3}$ S. Kamat, X. Su, R. Ballarini, and A. H. Heuer, "Structural basis for the fracture toughness of the shell of the conch Strombus gigas," Nature 405, 1036-1040 (2000).

${ }^{4}$ F. Vollrath, "Spider webs and silks," Sci. Am. 266, 70-76 (1992).

${ }^{5}$ J. M. Gosline, P. A. Guerette, C. S. Ortlepp, and K. N. Savage, "The mechanical design of spider silks: from fibroin sequence to mechanical function,” J. Exp. Biol. 202, 3295-3303 (1999).

${ }^{6} \mathrm{C}$. Boutry and T. Blackledge, "Biomechanical variation of silk links spinning plasticity to spider web function," Zoology 112, 451-460 (2009).

${ }^{7}$ A. Meyer, N. Pugno, and S. W. Cranford, "Compliant threads maximize spider silk connection strength and toughness," J. R. Soc., Interface 11, 20140561 (2014).

${ }^{8}$ S. Cranford, A. Tarakanova, N. Pugno, and M. Buehler, "Nonlinear material behaviour of spider silk yields robust webs," Nature 482, 72-76 (2012).

${ }^{9}$ R. Zaera, A. Soler, and J. Teus, "Uncovering changes in spider orb-web topology owing to aerodynamics effects," J. R. Soc., Interface 11, 20140484 (2014).

${ }^{10} \mathrm{Y}$. Aoyanagi and K. Okumura, "Simple model for the mechanics of spider webs," Phys. Rev. Lett. 104, 038102 (2010).
${ }^{11}$ M. S. Alam, M. A. Wahab, and C. H. Jenkins, "Mechanics in naturally compliant structures," Mech. Mater. 39, 145-160 (2007).

${ }^{12}$ F. K. Ko and J. Jovicic, "Modeling of mechanical properties and structural design of spider web," Biomacromolecules 5, 780-785 (2004).

${ }^{13}$ M. Miniaci, A. Krushynska, F. Bosia, and N. M. Pugno, "Large scale mechanical metamaterials as seismic shields," New J. Phys. (to be published).

${ }^{14}$ R. Martínez-Sala, J. Sancho, J. V. Sánchez, V. Gómez, J. Llinares, and F. Meseguer, "Sound attenuation by sculpture," Nature 378, 241 (1995).

${ }^{15}$ D. Bigoni, S. Guenneau, A. B. Movchan, and M. Brun, "Elastic metamaterials with inertial locally resonant structures: Application to lensing and localization," Phys. Rev. B 87, 174303 (2013).

${ }^{16}$ T. Zhu and E. Ertekin, "Phonon transport on two-dimensional graphene/ boron nitride superlattices," Phys. Rev. B 90, 195209 (2014).

${ }^{17}$ T. Zhu and E. Ertekin, "Resolving anomalous strain effects on twodimensional phonon flows: The cases of graphene, boron nitride, and planar superlattices," Phys. Rev. B 91, 205429 (2015).

${ }^{18}$ M. Fahrat, S. Enoch, S. Guenneau, and A. B. Movchan, "Broadband cylindrical acoustic cloak for linear surface waves in a fluid," Phys. Rev. Lett. 101, 134501 (2008).

${ }^{19} \mathrm{M}$. Maldovan, "Sound and heat revolutions in phononics," Nature 503, 209-217 (2013).

${ }^{20}$ L. Brillouin, Wave Propagation in Periodic Structures (McGraw-Hill Book Company, 1946).

${ }^{21}$ Z. Liu, X. Zhang, Y. Mao, Y. Y. Zhu, Z. Yang, C. T. Chan, and P. Sheng, "Locally resonant sonic materials," Science 289, 1734-1736 (2000).

${ }^{22}$ Y. Pennec, J. O. Vasseur, B. Djafari-Rouhani, L. Dobrzynski, and P. A. Deymier, "Two-dimensional phononic crystals: Examples and applications," Surf. Sci. Rep. 65, 229-291 (2010).

${ }^{23}$ M. I. Hussein, M. J. Leamy, and M. Ruzzene, "Dynamics of phononic materials and structures: Historical origins, recent progress, and future outlook," Appl. Mech. Rev. 66, 040802 (2014).

${ }^{24}$ A. O. Krushynska, V. G. Kouznetsova, and M. G. D. Geers, "Towards optimal design of locally resonant acoustic metamaterials," J. Mech. Phys. Solids 71, 179-196 (2014).

${ }^{25}$ M. Miniaci, A. Krushynska, F. Bosia, and N. M. Pugno, "Bio-inspired hierarchical dissipative metamaterials," e-print arXiv:1606.03596.

${ }^{26}$ P. Wang, F. Casadei, S. H. Kang, and K. Bertoldi, "Locally resonant band gaps in periodic beam lattices by tuning connectivity," Phys. Rev. B 91, 020103 (2015).

${ }^{27}$ Q. J. Lim, P. Wang, S. J. A. Koh, E. H. Khoo, and K. Bertoldi, "Wave propagation in fractal-inspired self-similar beam lattices," Appl. Phys. Lett. 107, 221911 (2015).

${ }^{28}$ P. G. Martinsson and A. B. Movchan, "Vibrations of lattice structures and phononic band gaps,” Q. J. Mech. Appl. Math. 56, 45-64 (2003).

${ }^{29}$ M. Miniaci, A. Marzani, N. Testoni, and L. De Marchi, "Complete band gaps in a polyvinyl chloride (PVC) phononic plate with cross-like holes: Numerical design and experimental verification," Ultrasonics 56, 251-259 (2015).

${ }^{30} \mathrm{P}$. Wang, J. Shim, and K. Bertoldi, "Effects of geometric and material nonlinearities on tunable band gaps and low-frequency directionality of phononic crystals," Phys. Rev. B 88, 014304 (2013).

${ }^{31}$ R. Sainidou, N. Stefanou, and A. Modinos, "Formation of absolute frequency gaps in three-dimensional solid phononic crystals," Phys. Rev. B 66, 212301 (2002).

${ }^{32}$ S. Timoshenko, Vibration Problems in Engineering, 3rd ed. (D. Van Nostrand Company Inc., Princeton, NJ, 1955). 


\section{Supplementary material for "Spider-Web Inspired Acoustic Metamaterials"}

Marco Miniaci ${ }^{1}$, Anastasiia Krushynska ${ }^{2}$, Alexander B. Movchan ${ }^{3}$, Federico Bosia ${ }^{2}$, Nicola M.

$$
\text { Pugno }{ }^{4,5,6 *}
$$

${ }^{1}$ University of Le Havre, Laboratoire Ondes et Milieux Complexes, UMR CNRS 6294,

76600 Le Havre, France

${ }^{2}$ Department of Physics, University of Turin, Via Pietro Giuria 1, 10125, Torino, Italy

${ }^{3}$ Department of Mathematical Sciences, University of Liverpool, L69 3BX, Liverpool, UK

${ }^{4}$ Laboratory of Bio-Inspired \& Graphene Nanomechanics, Department of Civil, Environmental and Mechanical Engineering, University of Trento, Via Mesiano, 77, 38123, Trento, Italy

${ }^{5}$ Center for Materials and Microsystems, Fondazione Bruno Kessler, Via Sommarive 18, 38123, Povo (Trento), Italy

${ }^{6}$ School of Engineering \& Materials Science, Queen Mary University of London, Mile End Road, London, E1 4NS, UK

* Corresponding authors: Federico.bosia@unito.it, nicola.pugno@unitn.it 


\section{A. Dispersion properties}

To investigate further details of the wave dispersion in proximity of BGs for the considered metamaterial configurations, we calculate group velocities $v_{g}=d \omega / d k$ and contour plots of dispersion surfaces for all directions of propagation. Figure S1 shows a schematic representation of the first irreducible Brillouin zone and Figure S2 shows the group velocity profiles for three spider-web inspired metamaterial configurations, the band structures of which are given in Figs. $2 \mathrm{~b}-\mathrm{d}$ of the main text. In particular, Fig. S2a provides $v_{g}$ curves along the $1^{\text {st }}$ irreducible Brillouin zone for a stiff frame and stiff ring resonators for $7^{\text {th }}-10^{\text {th }}$ pass bands. Figs. S2b and c show group velocities for the lower and upper bounds of the 1st BG for configurations with compliant ring resonators and (b) stiff or (c) compliant connectors joining the radial and circumferential ligaments.

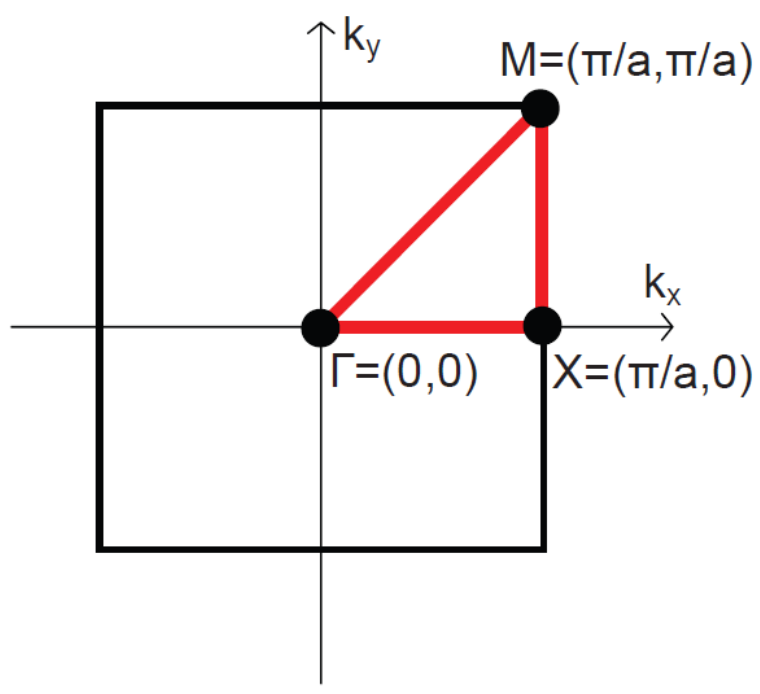

FIG. S1. $1^{\text {st }}$ irreducible Brillouin zone. 


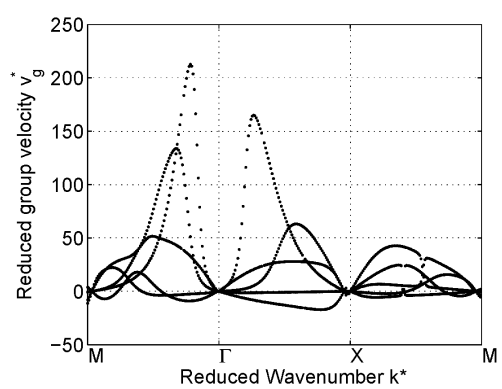

(a)

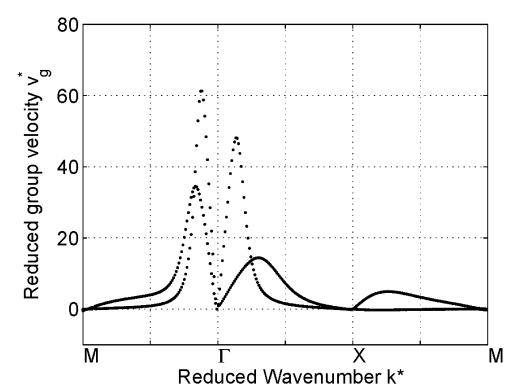

(b)

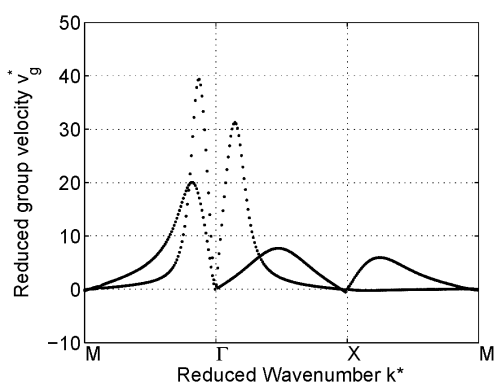

(c)

FIG. S2. Group velocity $v_{g}$ curves along the borders of the $1^{\text {st }}$ irreducible Brillouin zone for spider-web inspired lattices for (a) stiff frame and stiff ring resonators ( $7^{\text {th }}$ to $10^{\text {th }}$ curves) and compliant ring resonators with (b) stiff (bounds of the $1^{\text {st }} \mathrm{BG}$ ) and (c) compliant connectors joining the radial and circumferential ligaments (bounds of the $1^{\text {st }} \mathrm{BG}$ ).

Notice the presence of regions with negative group velocities in the $\Gamma-\mathrm{M}$ path and zero or almost zero group velocities (corresponding to localized modes) in proximity of the high symmetry points.

Finally, Fig. S3 shows the contour plots of dispersion surfaces corresponding to the branches below and above the first BGs presented in Fig. 2 b-d, from which it is possible to infer the potential of directionality of the considered system. Namely, the isofrequency contour plots for some modes at specific frequencies appears as approximately circular, which suggests a quasi isotropic behaviour for that frequency range. In contrast dispersion surfaces for other modes, or for the same mode but at different frequencies appear mostly aligned along preferential directions (namely $x$ and $y$ ), suggesting a very anisotropic behaviour in the system. 

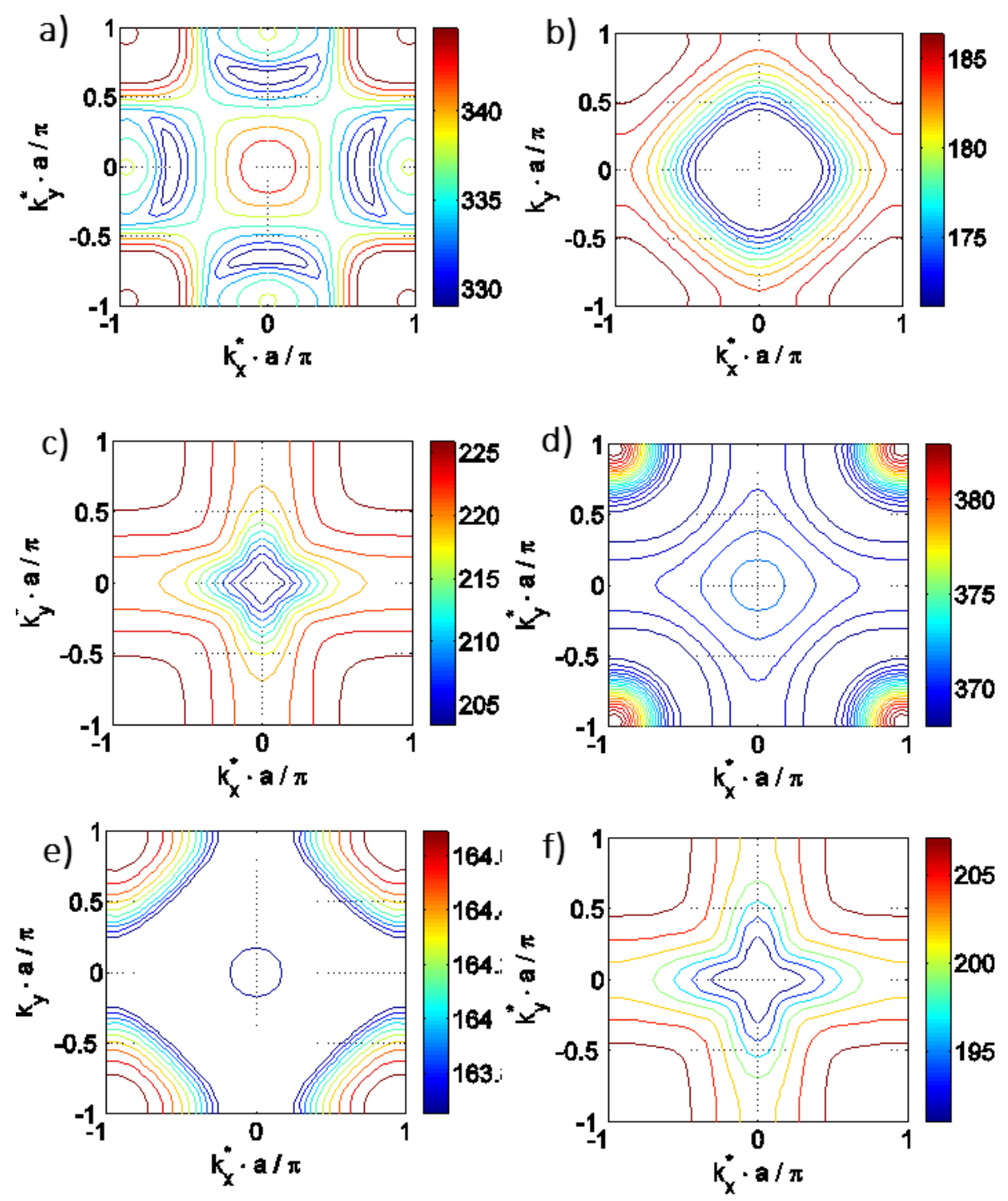

FIG. S3. Contour plots for dispersion surfaces corresponding to the branches below and above the first BGs presented in Fig. 2 b-d (main text). 


\section{B. Dependence on number of ring resonators}

We now investigate the evolution of the analyzed band structures by varying the number of ring resonators. Fig. S4 shows dispersion diagrams for the unit cells with 1, 3, 5 and 7 stiff ring resonators (dragline silk). In all cases, the unit cells with a reduced number of resonators are obtained by removing the most internal resonators from the original geometry shown (Fig. 1b). Stiff rings are chosen here, since the corresponding band structures have fewer pass bands, thus facilitating the analysis. In the considered frequency range, the number of localized modes decreases with the increase of the number of the ring resonators, since the structure becomes stiffer. This results in an increase in the number of band gaps, which are also shifted to lower frequencies, since the wave attenuation is enhanced due to increased number of resonators. Interestingly, the unit cells with $3-7$ ring resonators possess the first (narrow) band gap at the same frequencies, slightly above $150 \mathrm{~Hz}$. Examination of the corresponding vibration forms reveals that this band gap is induced by local resonances in the external square frame. Thus, the gap frequencies are independent of the number of the ring resonators, as well as of their mechanical properties (Fig. S4)

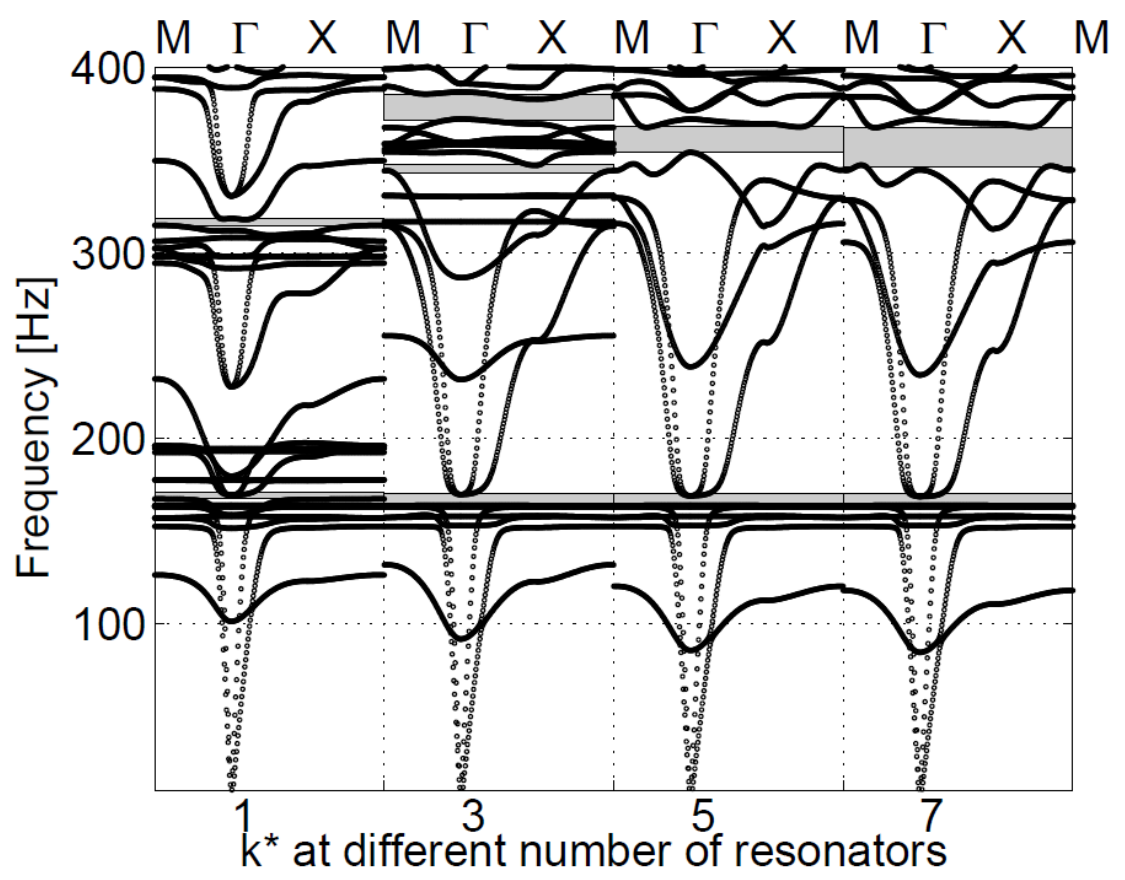


FIG. S4. Band structures for the spider web-inspired unit cells comprising 1, 3, 5 and 7 outer ring resonators.

\section{Natural frequencies of ring resonators}

As mentioned in the main text, vibrations corresponding to an important class of standing waves are associated with the ring resonators, whose natural frequencies $\omega_{n}$ for non-axisymmetric in-plane flexural vibrations are known [1,2] in closed form, see Eq.(1). In this section, we analyse whether these frequencies can be used to evaluate the frequencies of localized modes or band gap bounds in the spider-web inspired lattice.

For this purpose, we consider a single ring resonator, e.g. a $2^{\text {nd }}$ external resonator in the spider-web-inspired unit cell shown in Fig. 1b. The first 4 natural frequencies of this resonator occur at $10.7 \mathrm{~Hz}, 30.2 \mathrm{~Hz}, 57.8 \mathrm{~Hz}$, and $93.5 \mathrm{~Hz}$, according to Eq. (1). In order to compare these frequencies and the corresponding vibration forms with those found in the band structure of the spider-web inspired lattice, we assign the mechanical properties given in Table S1 to the spider-web inspired unit cells. We then consider various cases of modified values of the Young's modulus as given in Table $\mathrm{S} 2$.

The comparison of analytically-evaluated natural frequencies with those obtained numerically for the study cases 1-5 indicated in Table S2 is given in Figure S5. As can be seen, non-zero values of the mechanical parameters for soft radial silk (as compared to zero values implied in the analytical solution) result in a coupling between the vibrations of individual ring resonators with the bearing frame. This coupling results in an increase of eigenfrequencies of ring resonators and makes analytical solutions not applicable to the evaluation of the frequencies of localized modes in the spider-web inspired lattice. The vibration forms corresponding to the analysed study cases are given in Figures S6-S9. 
Table S1. Material parameters for constituents of the spider-web inspired unit cell. Indicated values are assigned to highlighted (blue) parts of the unit cell.

\begin{tabular}{|c|c|}
\hline Viscid silk & Stiff radial silk radial silk \\
$\rho_{v}=1200 \mathrm{~kg} / \mathrm{m}^{3}$ & $E_{r}^{s t}=1200 \mathrm{GPa}$ \\
$E_{v}=1.2 \mathrm{GPa}$ & $\rho_{r}^{s t}=1200 \mathrm{~kg} / \mathrm{m}^{3}$
\end{tabular}

Table S2. The case studies with various material parameters for the soft radial silk.

\begin{tabular}{|c|c|c|c|}
\hline Study case N. & $E_{r}^{s o}[\mathrm{~Pa}]$ & $\rho_{r}^{s o}\left[\mathrm{~kg} / \mathrm{m}^{3}\right]$ & Note \\
\hline Case 0 & 0 & 0 & Analytical solution \\
\hline Case 1 & $1.2 \times 10^{3}$ & 1.2 & $E_{r}^{s o} / \rho_{r}^{s o}=10^{3}$ \\
\hline Case 2 & $1.2 \times 10^{4}$ & 12 & $E_{r}^{s o} / \rho_{r}^{s o}=10^{3}$ \\
\hline Case 3 & $1.2 \times 10^{5}$ & 120 & $E_{r}^{s o} / \rho_{r}^{s o}=10^{3}$ \\
\hline Case 4 & $1.2 \times 10^{6}$ & 1200 & $E_{r}^{s o} / \rho_{r}^{s o}=10^{3}$ \\
\hline Case 5 & $1.2 \times 10^{7}$ & 1200 & $E_{r}^{s o} / \rho_{r}^{s o}=10^{4}$ \\
\hline
\end{tabular}




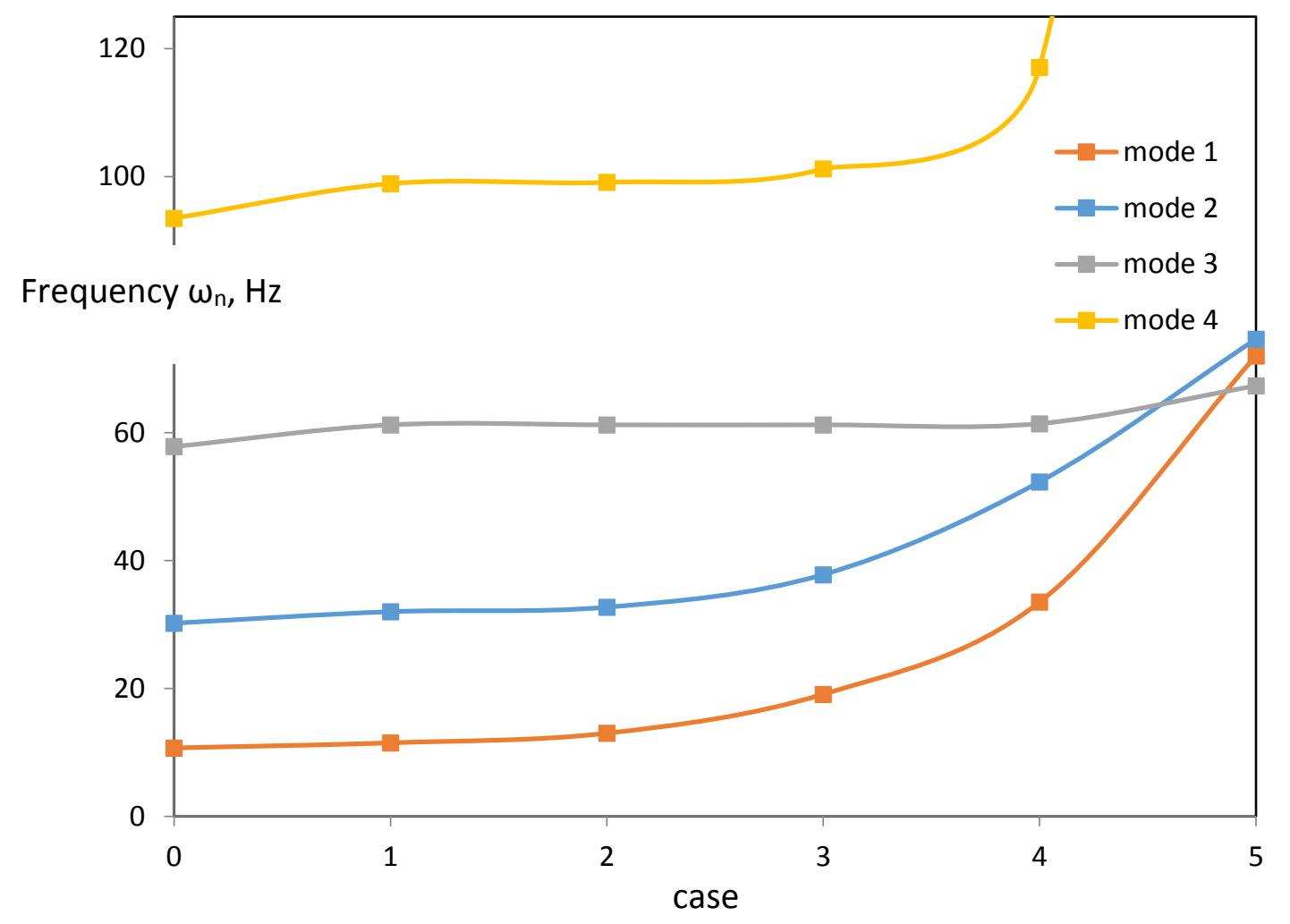

Figure S5. Analytically and numerically evaluated frequencies of the first 4 modes of the 2nd external resonator in the spider-web inspired unit cell (Fig. 1b). The numbering of the study cases corresponds to that given in Table S2. 


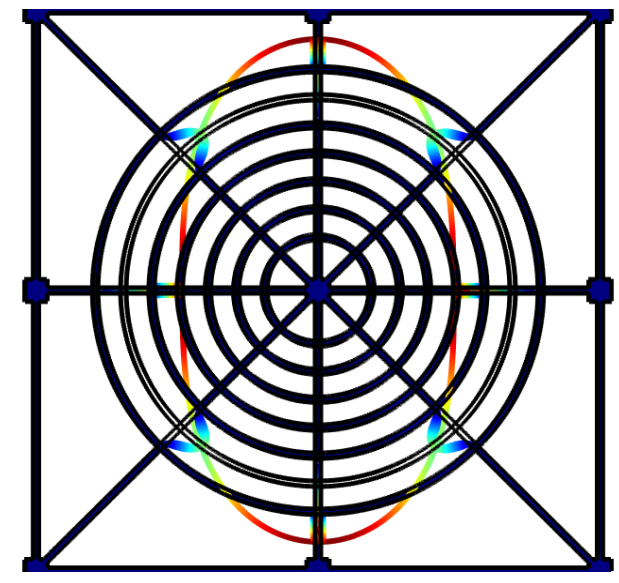

(a) Case 1, $11.5 \mathrm{~Hz}$

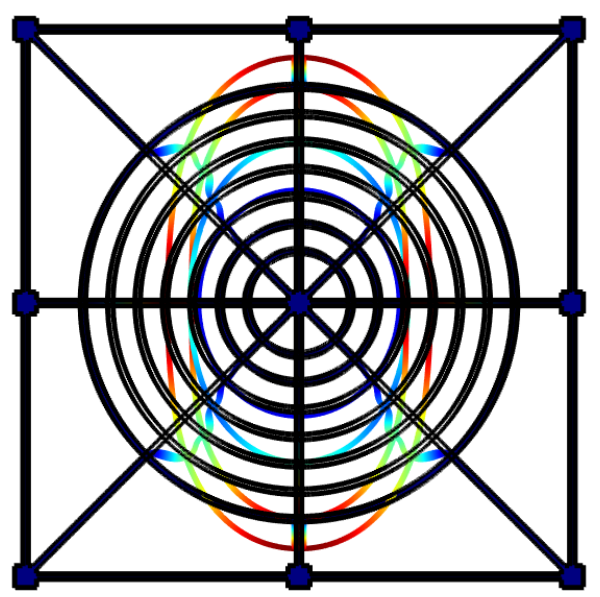

(c) Case 3, $19.1 \mathrm{~Hz}$

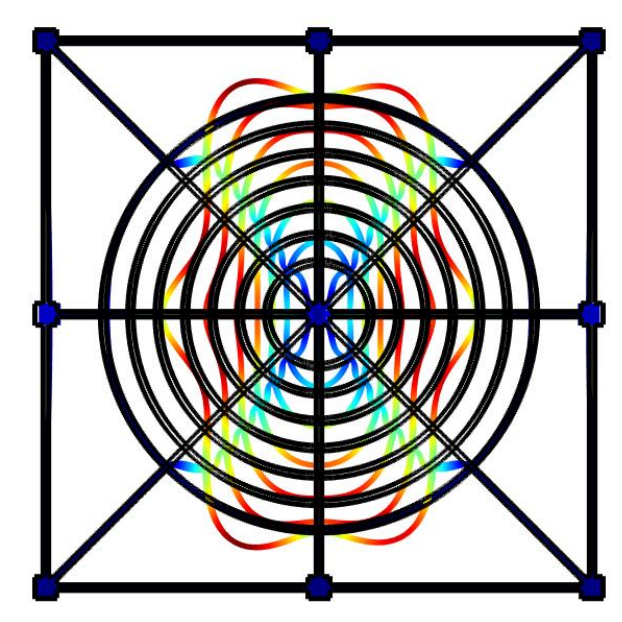

(e) Case $5,72.0 \mathrm{~Hz}$

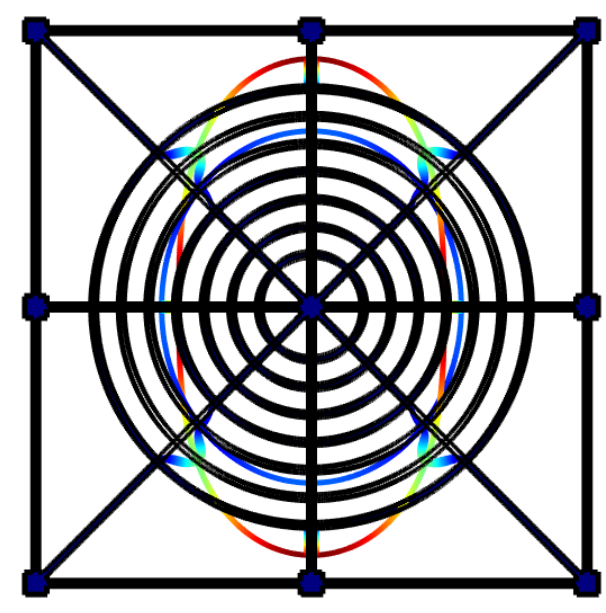

(b) Case 2, $13.0 \mathrm{~Hz}$

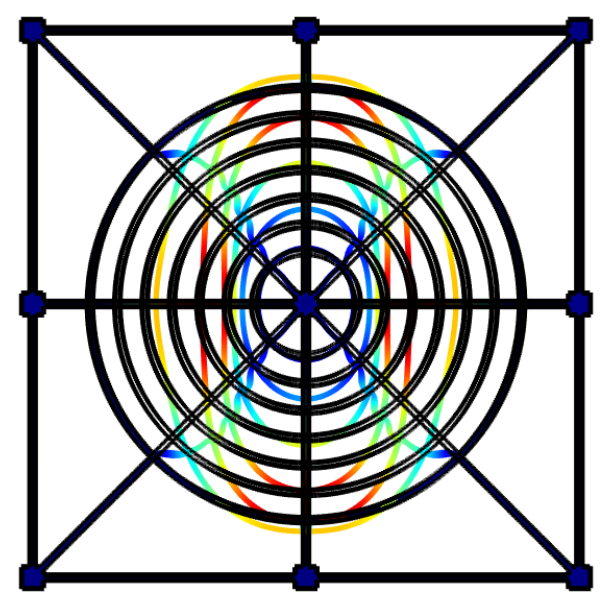

(d) Case 4, 33.5 Hz

(d) Case 4, 33.5 Hz

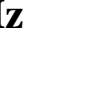




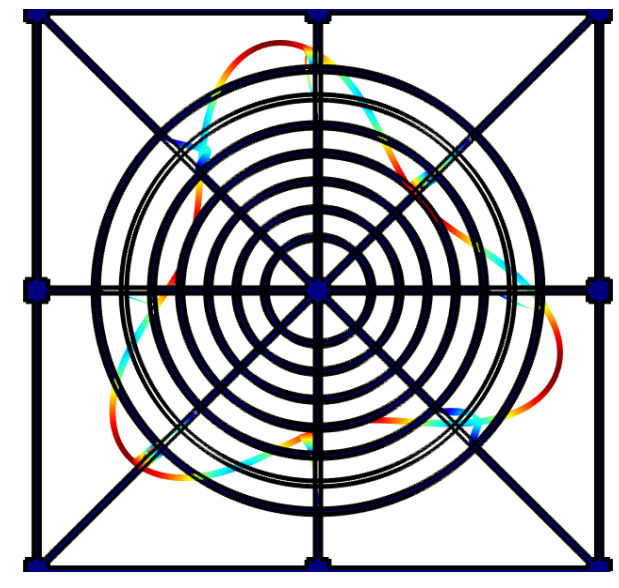

(a) Case 1, 32.0 Hz

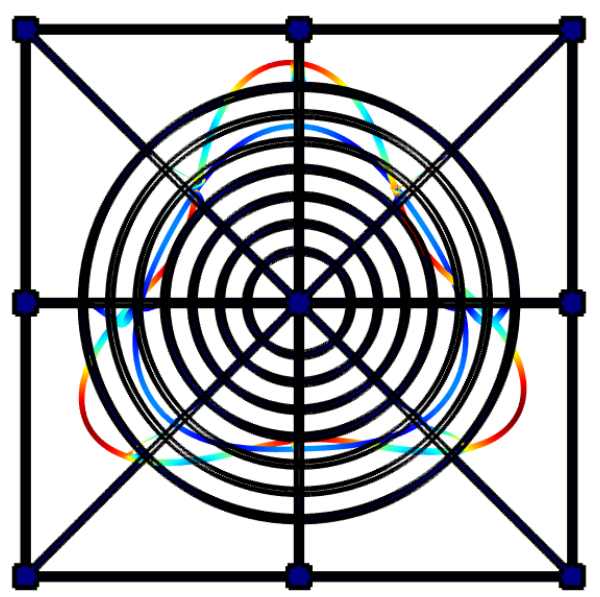

(c) Case 3, 37.8 Hz

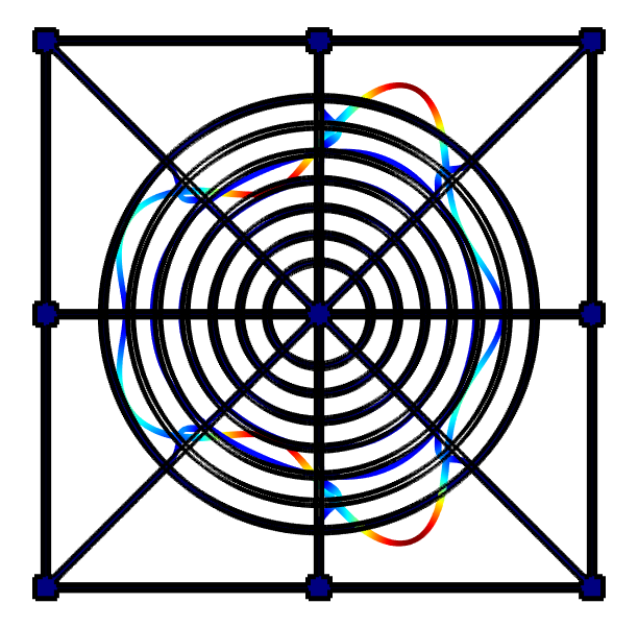

(e) Case 5, 74.0 Hz

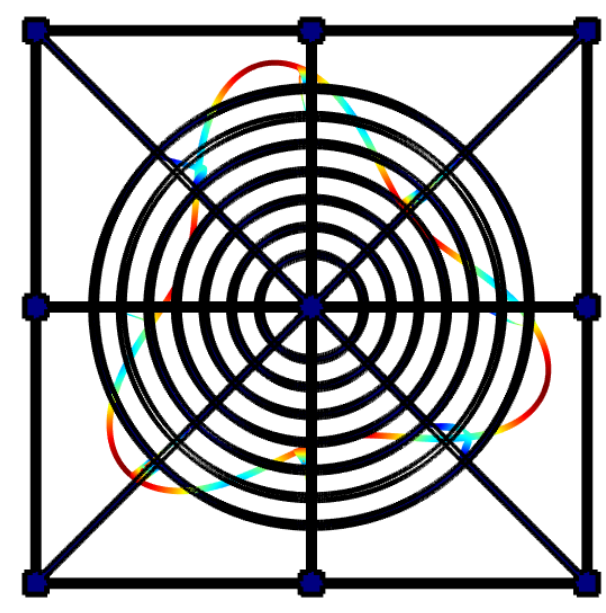

(b) Case 2, $32.7 \mathrm{~Hz}$

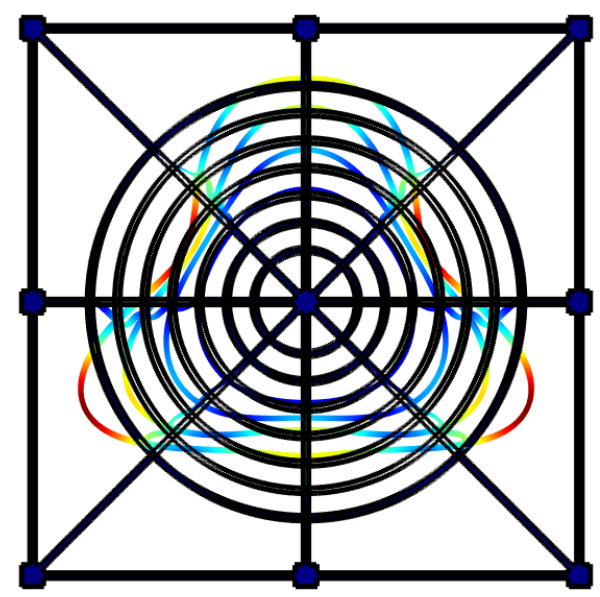

(d) Case 4, 52.3 Hz

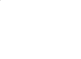




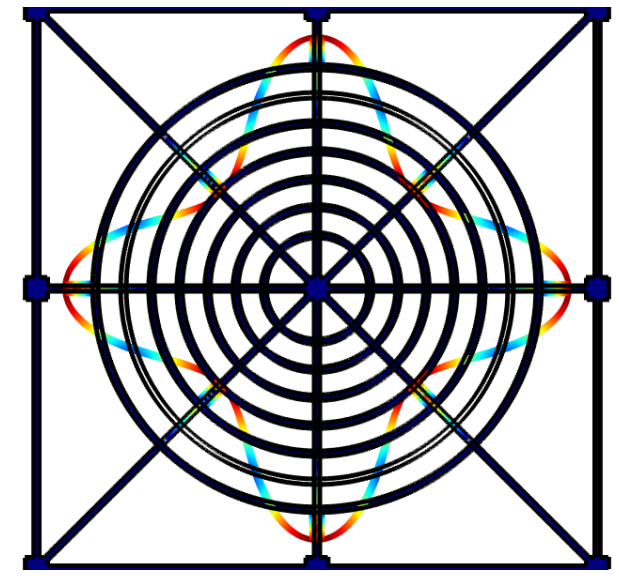

(a) Case 1, 61.2 Hz

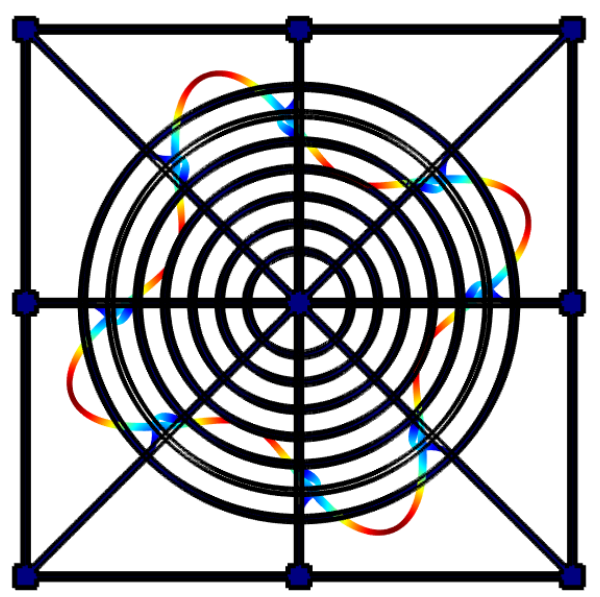

(c) Case 3, 61.2 Hz

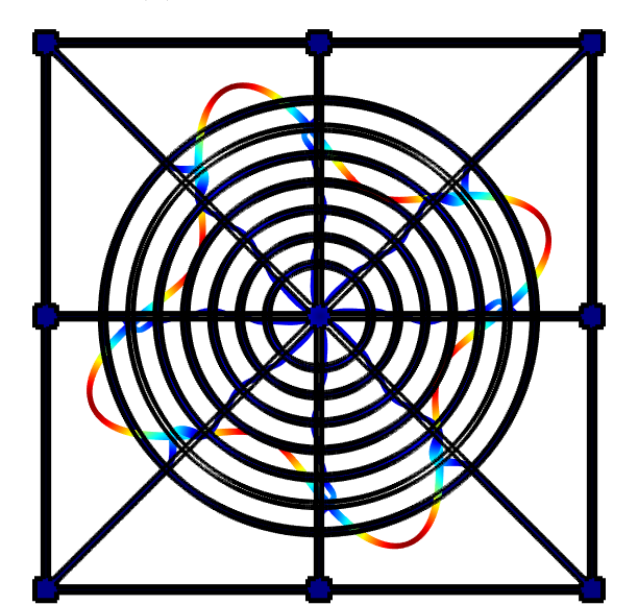

(e) Case 5, 67.3 Hz

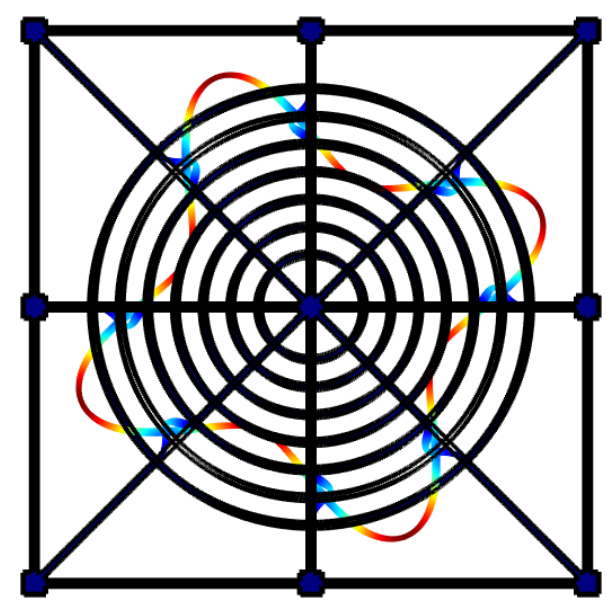

(b) Case 2, 61.2 Hz

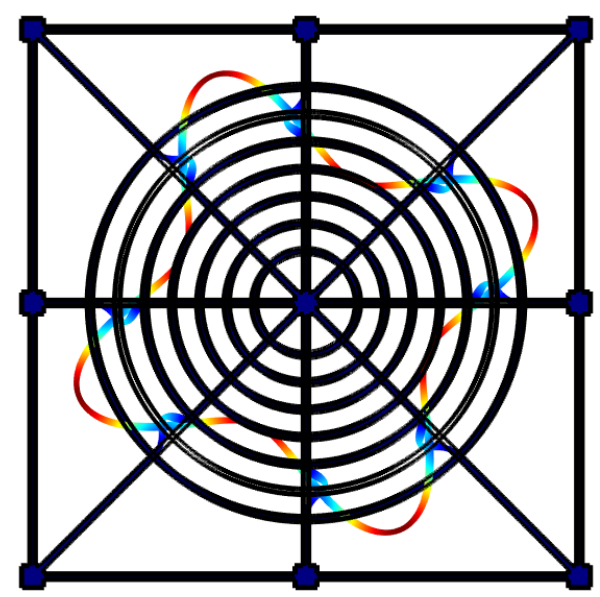

(d) Case 4, 61.4 Hz

Figure S8. Vibration patterns of the $\mathbf{3}^{\text {rd }}$ mode in the spider-web inspired unit cell (Fig. 1b). The numbering of the study cases corresponds to that given in Table S2. 


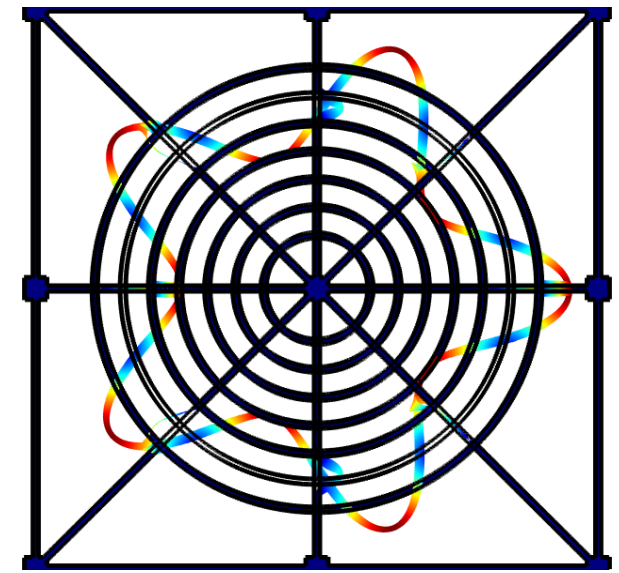

(a) Case $1,98.9 \mathrm{~Hz}$

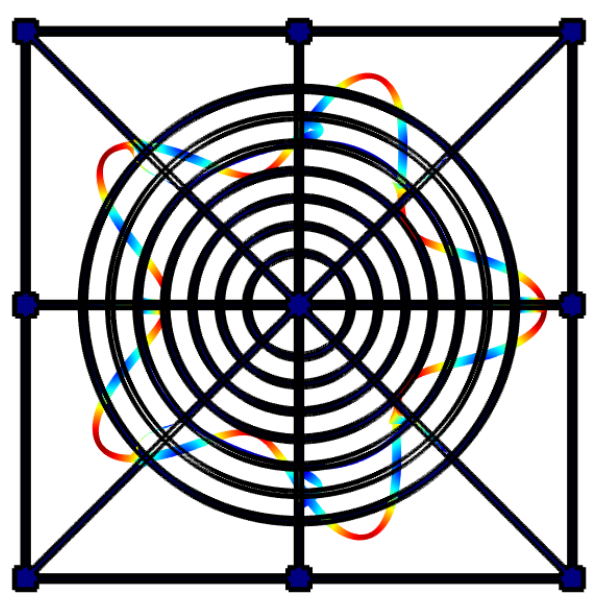

(c) Case 3, 101.2 Hz

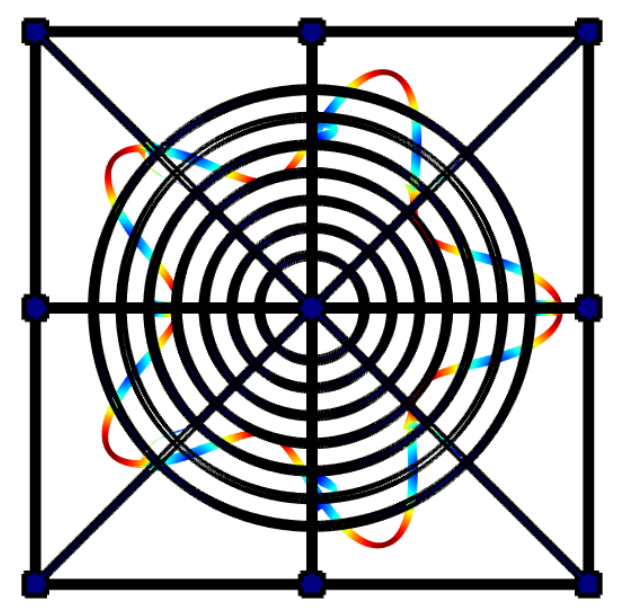

(b) Case 2, $99.1 \mathrm{~Hz}$

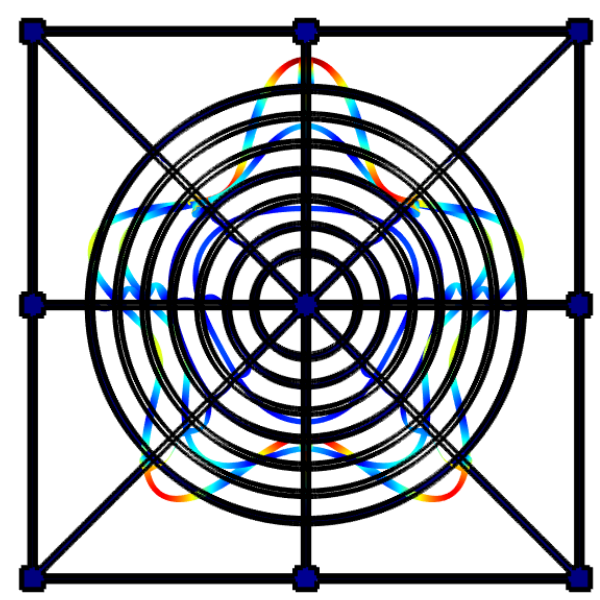

(d) Case 4, 117.1 Hz

Figure S9. Vibration patterns of the $4^{\text {th }}$ mode in the spider-web inspired unit cell (Fig. 1b). The numbering of the study cases corresponds to that given in Table S2.

\section{Analysis of the vibration patterns}

This section presents an analysis of the evolution of the dynamics for individual modes as the metamaterial geometry is changed from a regular lattice to the spider-web inspired lattice.

The consideration of the spider-web lattice is performed for the two cases mentioned in the main text, namely when both the circumferential ligaments and their connectors have either stiff (dragline silk) or compliant (viscid silk) mechanical properties. 


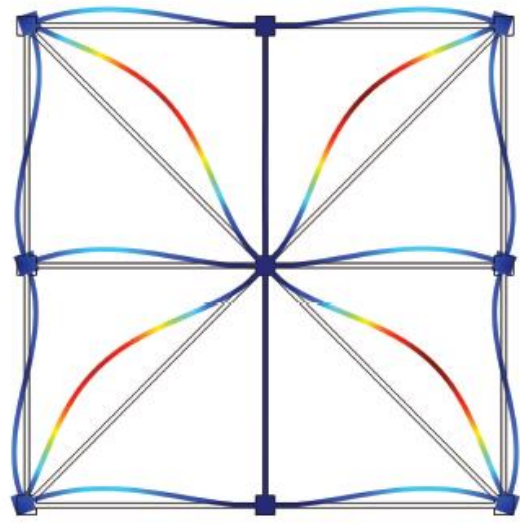

(a)

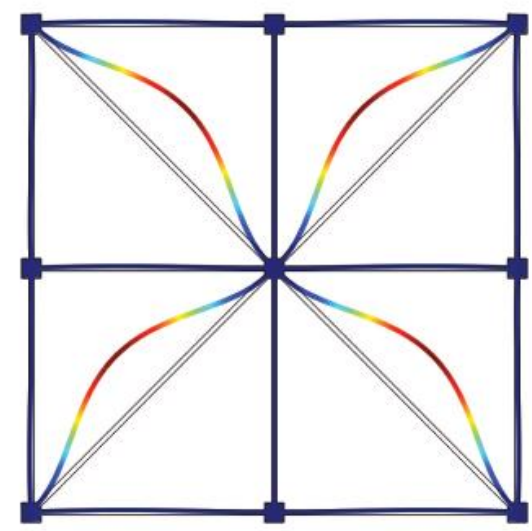

(d)

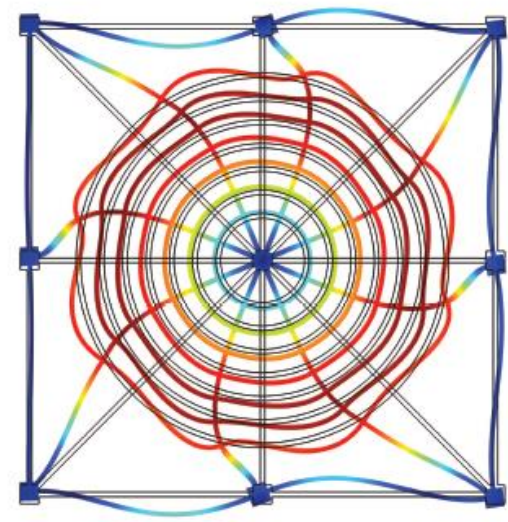

(b)

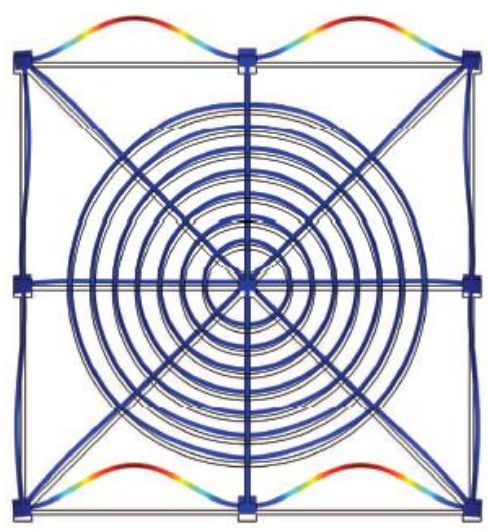

(e)

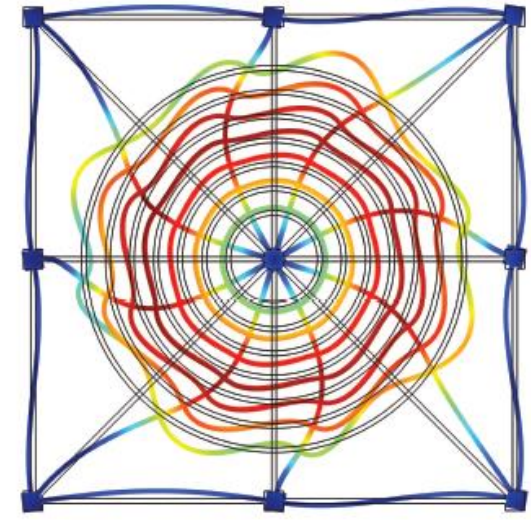

(c)

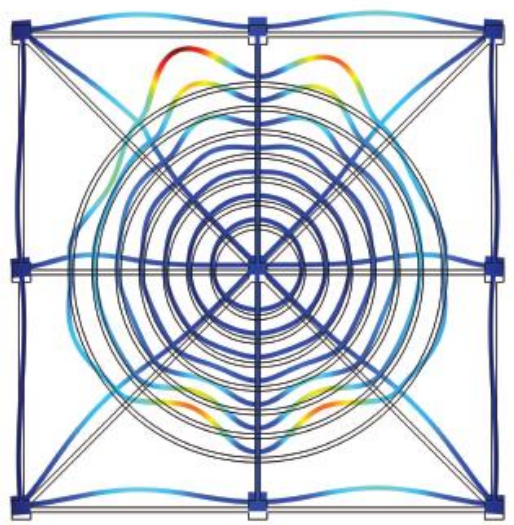

(f)

Figure S10. The Bloch modes of the (a-c) $1^{\text {st }}$ and (d-f) $2^{\text {nd }}$ propagation modes for the 3 case studies analysed in the main text: (a,d) regular lattice, spider-web lattices with (b,e) dragline silk or (c,f) viscid silk material properties for the ring resonators. Here, $\mathbf{k}=(7 \pi / 10 a, 0)$ is associated to (a) $\mathrm{f}$ $=61 \mathrm{~Hz},(\mathrm{~b}) \mathrm{f}=107 \mathrm{~Hz}$, (c) $\mathrm{f}=63 \mathrm{~Hz}$ and $\mathbf{k}=(\pi / 2 a, 0)$ is associated to (d) $\mathrm{f}=77 \mathrm{~Hz},(\mathrm{e}) \mathrm{f}=$ $144 \mathrm{~Hz}$, (f) $\mathrm{f}=117 \mathrm{~Hz}$, respectively. The magnitudes of the modal displacements are shown with maximum and minimum values indicated by red and blue, respectively.

As can be seen in Fig. 2 of the main text, the frequencies of the $1^{\text {st }}$ (localized) mode (the first curve from the bottom) are the same for the regular and spider-web inspired lattices, except for the case shown in Fig. 2b. This occurs due to the fact that for this mode, it is mainly radial ligaments that are involved into the motion (Figs. S10 a-c). The addition of compliant circumferential 
ligaments (Fig. S10c) only slightly changes the mode frequency (from $61 \mathrm{~Hz}$ for the regular lattice to $63 \mathrm{~Hz}$ for the spider-web lattice), while the rotational nature of the mode is preserved. On the contrary, stiff circumferential ligaments (Fig. S10b) with larger inertia significantly influence the frequency value $(107 \mathrm{~Hz}$ for the spider-web lattice vs. $61 \mathrm{~Hz}$ for the regular lattice), while the vibration form remains almost unchanged (Fig. S6b).

The frequency of the $2^{\text {nd }}$ mode in Fig. 2 of the main text (second curve from the bottom) is shifted to higher frequencies as circumferential ligaments are added, regardless of their mechanical properties. Examination of the corresponding vibration patterns in Fig. S10 d-f reveals that in the regular lattice, radial ligaments vibrate independently from each other. The introduction of circumferential ligaments results in interactions that lead to a stiffer overall structure and the dynamics of the second mode changes completely. If stiff mechanical properties are assumed, the primary framework vibrates, while the internal part is almost motionless (Fig.S10e). If resonators have compliant mechanical properties with respect to the connectors, some localized motions arise (Fig.S10f). However, in both cases the central part of the systems experiences less motion compared to the external part.

Inspection of the other modes reveals that it is possible to achieve strong localization of displacements either in the external frame (Figs. S11a-b) or inside the resonators (Figs. S11c-d) regardless of the resonator mechanical properties. Also, as the frequency increases, more resonators are involved in the vibration, since the inner resonators also become excited (Figs. S11c-d).

Overall, some of the localized modes exhibit mainly rotational displacements confined to the internal portion of the unit cell. Since these modes are weakly coupled to the propagating shear and pressure waves in the external frame, they do not open BGs. Instead, other modes characterized by interactions between resonators and radial ligaments generate complete band gaps highlighted in grey in Fig. 2. Although some anisotropy may be present in the modes, these band gaps are omnidirectional. 


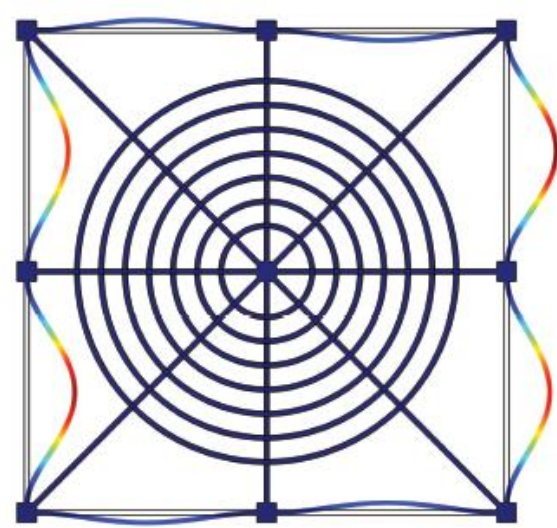

(a)

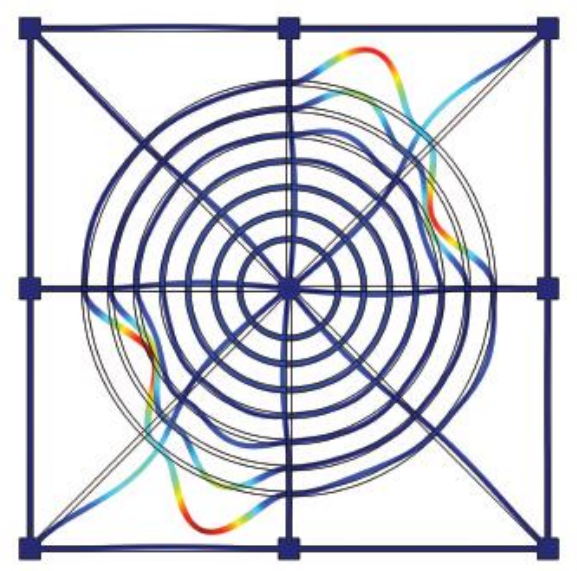

(c)

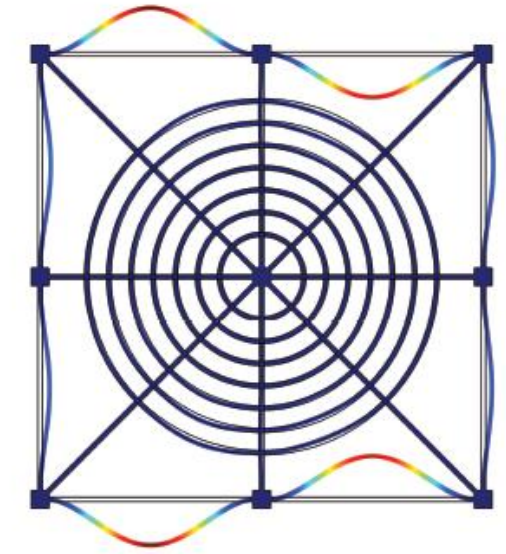

(b)

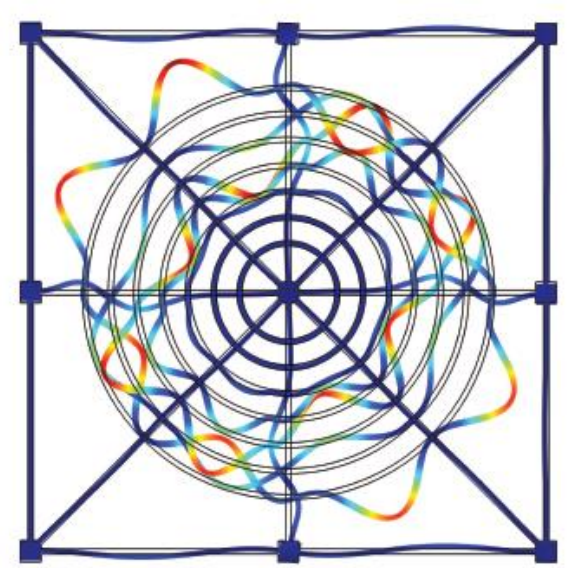

(d)

Figure S11. The Bloch modes with vibrations localized in the external frame for the spider-web inspired unit cells, where resonators have (a) the same mechanical properties as the connectors and (b) different mechanical properties from the connectors. The Bloch modes showing an increased number of resonators involved in the motion as the frequency increases from (c) $\mathrm{f}=144 \mathrm{~Hz}$ to (d) $\mathrm{f}$ $=261 \mathrm{~Hz}$.

\section{References}

[1] S. Timoshenko, Vibration problems in engineering, Princeton: D. Van Nostrand Company, 1955.

[2] A. E. H. Love, A treatise on the mathematical theory of elasticity, New York: Dover Publications, 1944. 